\title{
Cold Exposure Drives Weight Gain and Adiposity following Chronic Suppression of Brown Adipose Tissue
}

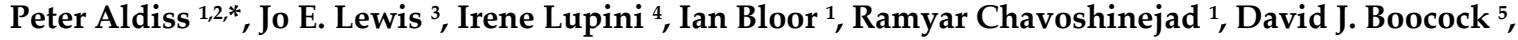 \\ Amanda K. Miles ${ }^{5}$, Francis J. P. Ebling ${ }^{3}$, Helen Budge ${ }^{1}$ and Michael E. Symonds ${ }^{1,6, *}$
}

Citation: Aldiss, P.; Lewis, J.E.; Lupini, I.; Bloor, I.; Chavoshinejad, R.; Boocock, D.J.; Miles, A.K.; Ebling, F.J.P.; Budge, H.; Symonds, M.E. Cold Exposure Drives Weight Gain and Adiposity Following Chronic Suppression of Brown Adipose Tissue. Int. J. Mol. Sci. 2022, 23, 1869. https://doi.org/10.3390/ijms23031869 Academic Editor: Jean-françois Tanti Received: 9 November 2021 Accepted: 20 January 2022 Published: 7 February 2022

Publisher's Note: MDPI stays neutral with regard to jurisdictional claims in published maps and institutional affiliations.

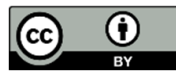

Copyright: @ 2022 by the authors. Licensee MDPI, Basel, Switzerland. This article is an open access article distributed under the terms and conditions of the Creative Commons Attribution (CC BY) license (https://creativecommons.org/licenses/by/4.0/).
1 Academic Unit of Population and Lifespan Sciences, Centre for Perinatal Research, School of Medicine, University of Nottingham, Nottingham NG7 2UH, UK; ian.bloor@nottingham.ac.uk (I.B.); ramyar.chavoshinejad@nottingham.ac.uk (R.C.); helen.budge@nottingham.ac.uk (H.B.)

2 Section for Nutrient and Metabolite Sensing, The Novo Nordisk Foundation Center for Basic Metabolic Research, University of Copenhagen, 2200 Copenhagen, Denmark

3 Queen's Medical Centre, School of Life Sciences, University of Nottingham, Nottingham NG11 8N, UK; j12033@medschl.cam.ac.uk (J.E.L.); fran.ebling@nottingham.ac.uk (F.J.P.E.)

4 School of Biosciences and Veterinary Medicine, University of Camerino, 62032 Camerino, Italy; Irene.lupini@studenti.unicam.it

5 John van Geest Cancer Research Centre, Nottingham Trent University, Nottingham NG11 8N, UK; david.boocock@ntu.ac.uk (D.J.B.); amanda.miles@ntu.ac.uk (A.K.M.)

6 Nottingham Digestive Disease Centre, Biomedical Research Center, School of Medicine, University of Nottingham, Nottingham NG11 8N, UK

* Correspondence: Peter.aldiss@sund.ku.dk (P.A.); michael.symonds@nottingham.ac.uk (M.E.S.)

\begin{abstract}
Therapeutic activation of thermogenic brown adipose tissue (BAT) may be feasible to prevent, or treat, cardiometabolic disease. However, rodents are commonly housed below thermoneutrality $\left(20^{\circ} \mathrm{C}\right)$ which can modulate their metabolism and physiology including the hyperactivation of brown (BAT) and beige white adipose tissue. We housed animals at thermoneutrality from weaning to chronically supress BAT, mimic human physiology and explore the efficacy of chronic, mild cold exposure $\left(20^{\circ} \mathrm{C}\right)$ and $\beta 3$-adrenoreceptor agonism (YM-178) under these conditions. Using metabolic phenotyping and exploratory proteomics we show that transfer from $28{ }^{\circ} \mathrm{C}$ to $20{ }^{\circ} \mathrm{C}$ drives weight gain and a $125 \%$ increase in subcutaneous fat mass, an effect not seen with YM-178 administration, thus suggesting a direct effect of a cool ambient temperature in promoting weight gain and further adiposity in obese rats. Following chronic suppression of BAT, uncoupling protein 1 mRNA was undetectable in the subcutaneous inguinal white adipose tissue (IWAT) in all groups. Using exploratory adipose tissue proteomics, we reveal novel gene ontology terms associated with cold-induced weight gain in BAT and IWAT whilst Reactome pathway analysis highlights the regulation of mitotic (i.e., G2/M transition) and metabolism of amino acids and derivatives pathways. Conversely, YM-178 had minimal metabolic-related effects but modified pathways involved in proteolysis (i.e., eukaryotic translation initiation) and RNA surveillance across both tissues. Taken together these findings are indicative of a novel mechanism whereby animals increase body weight and fat mass following chronic suppression of adaptive thermogenesis from weaning. In addition, treatment with a B3-adrenoreceptor agonist did not improve metabolic health in obese animals raised at thermoneutrality.
\end{abstract}

Keywords: brown adipose tissue; thermoneutrality; healthy expansion of adipose tissue; proteomics

\section{Introduction}

Therapeutic activation of thermogenic brown adipose tissue (BAT) may be feasible to prevent, or treat, cardiometabolic disease [1]. In rodent models of obesity, the activation of BAT and uncoupling protein (UCP1)-positive beige adipocytes in white adipose tissue 
(WAT) by cold exposure and sympathomimetics (i.e., $\beta 3$-agonists) can attenuate or reverse obesity, diabetes and atherosclerosis, thus improving metabolic health [1]. A major factor influencing these outcomes is that animals are typically housed at temperatures well below their thermoneutral zone (which for a rodent is c. $28^{\circ} \mathrm{C}$ ) [2]. Under these conditions, not only is BAT hyperactive, but $\mathrm{UCP} 1+$ beige adipocytes are readily seen in the inguinal WAT (IWAT) depot [3].

The 'cold-stressed' animal has been widely studied but much less is known about the underlying adaptations in adipose tissue of animals maintained at thermoneutrality. Usually, temperatures as low as $4{ }^{\circ} \mathrm{C}$, which represent an 'extreme cold', are used to activate BAT, with the induction of UCP1 and subsequent thermogenic response primarily seen in subcutaneous IWAT and other 'beige' depots [3]. Conversely, when animals are housed at thermoneutrality and then exposed to $20^{\circ} \mathrm{C}$, the induction of $\mathrm{UCP} 1$ is primarily seen in BAT. These differences suggest there are two steps to the 'browning' process and emphasise the need to study rodent metabolism under thermoneutral conditions. Importantly, it was recently demonstrated that BAT from obese mice, housed chronically at thermoneutrality closely resembles human BAT [4]. This model of 'physiologically humanised BAT' is now thought to represent the best choice for studying the physiology of this key metabolic tissue and it has recently been shown that intermittent cold exposure of thermoneutrally housed animals exacerbates diet-induced obesity [5]. Here, we extend this recent work by raising animals at thermoneutrality, on an obesogenic diet from weaning. Beginning this early is a particularly important consideration given the early developmental steps which would be occurring in early life [6]. Furthermore, we use rats as classically, their physiology to external stressors such as diet and the environment is closer, than mice, to humans [7]. Using this model we have demonstrated UCP1 mRNA is absent in subcutaneous IWAT (the classical 'beige' depot) and not induced with exercise training [8], a common response at standard housing temperatures and one which is typically not seen in humans [9]. We hypothesised that activation of BAT by mild cold and YM-178, a highly selective $\beta 3$-agonist that was recently been shown to activate BAT in lean and obese humans, and drive improvements in lipid metabolism, would be negligible under these conditions [10,11]. Moreover, we hypothesised that perivascular BAT (PVAT) which surrounds the thoracic aorta and plays a crucial role in vascular function, lipid metabolism and thermoregulation would be more responsive, as was previously shown to be the case with exercise training $[8,12,13]$. Finally, we sought to determine how the AT proteome responds following chronic suppression of BAT to better understand the molecular response to potentially thermogenic stimuli at thermoneutrality.

\section{Results}

Therapeutic activation of BAT by cold exposure drives weight loss and improves metabolic health. However, basal BAT activity is an undoubtedly an important consideration in the interpretation and translation of this field to humans. Animals housed at standard housing temperatures (i.e., $20-22^{\circ} \mathrm{C}$ ) have chronically active BAT, which exhibits supra-physiologically activity when animals are cold-exposed (i.e., to $4{ }^{\circ} \mathrm{C}$ ).

Modelling human physiology to 'humanise' BAT we raised animals at thermoneutrality (i.e., $28^{\circ} \mathrm{C}$ ) on high-fat diet from weaning to 12 weeks old. Four weeks of subsequent exposure to mild cold (i.e., $20^{\circ} \mathrm{C}$ ) promoted weight gain and increased BAT and subcutaneous IWAT mass, an effect not seen with a clinically relevant dose of the highly selective $\beta 3$-agonist YM-178 (Figures 1 and 2A-E) [14]. There was no detectable change in total fat mass (i.e., the total of all dissected depots), gonadal (GWAT), mesenteric (MWAT), retroperitoneal (RPWAT) or paracardial (PCAT) fat depots, or in liver or heart mass (Figures 2C and S1B-G) suggesting cold does not drive whole-body changes in adiposity, or lean mass. There was however a significant increase in kidney size (Figure S1H). As there was no difference in $24 \mathrm{~h}$ energy expenditure, or intake, (Figure S1I,J) we incorporated these parameters, alongside body mass, into an ANCOVA. Whilst there was no evidence that changes in body mass were associated with altered energy expenditure 
(Figure 2G) in cold exposed rats there was a significant, unexpected relationship between body mass and energy intake in this group $\left(\mathrm{r}^{2}=0.852, p=0.025\right.$, Figure $\left.2 \mathrm{H}\right)$ which was not seen in animals treated with YM-178. (Figure $2 \mathrm{~A}-\mathrm{H}$ ). Increased weight gain and adiposity in cold-exposed animals was not associated with impaired metabolic parameters (i.e., serum glucose, triglycerides and NEFA) or hormones (i.e., insulin and leptin) (Figure 2I-N) suggesting weight gain was not pathological whilst there was no difference in corticosterone (Figure S1K) between groups suggesting no impact of cold exposure, or YM178 , on chronic stress.

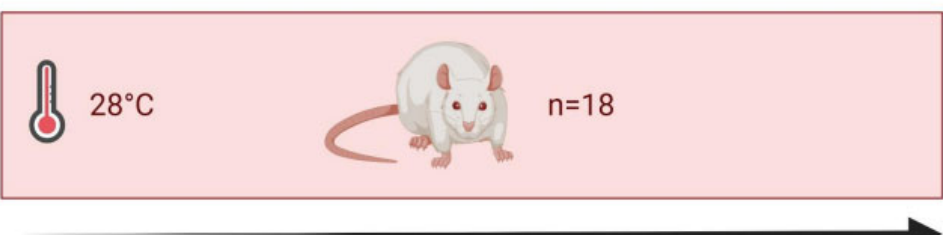

3-12 weeks old
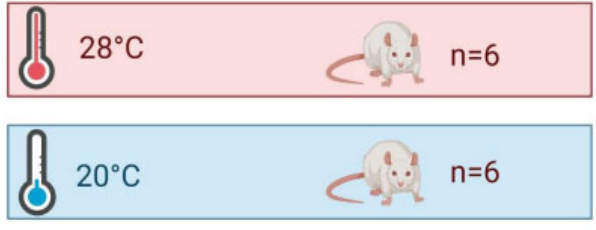

$\left\{\begin{array}{c}28^{\circ} \mathrm{C}+\beta 3 \\ \text { agonist }\end{array}\right.$

12-16 weeks old

Figure 1. Experimental outline for study.
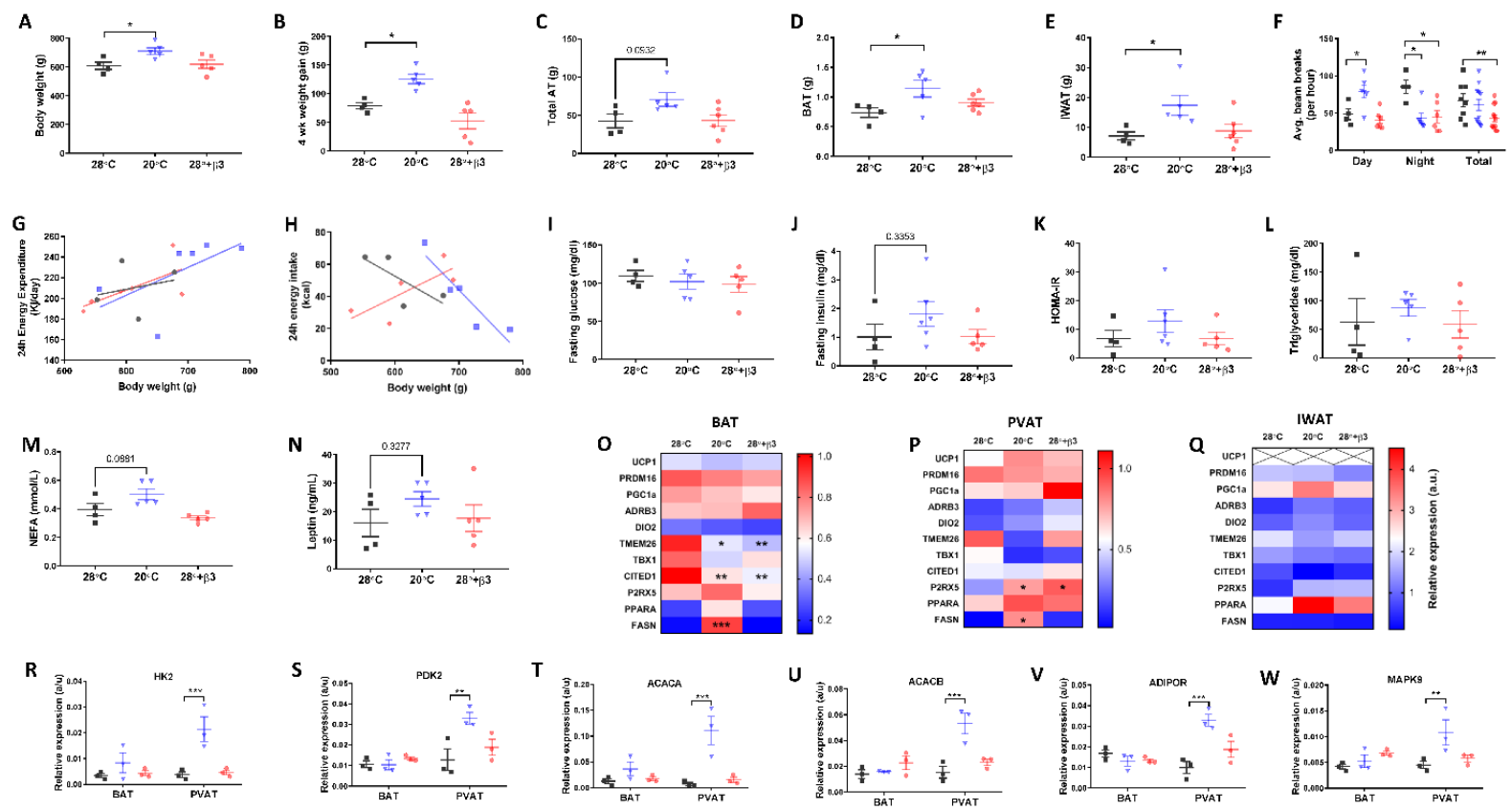

Figure 2. Cold exposure $\left(20^{\circ} \mathrm{C}\right)$ but not $\mathrm{YM}-178\left(28^{\circ} \mathrm{C}+\beta 3\right)$ drove weight gain and deposition of BAT and inguinal white adipose tissue (IWAT) with no effect on serum metabolites or thermogenic markers. (A) Final body weight, (B) 4-week intervention weight gain, (C) total fat mass, (D) BAT mass, (E) IWAT mass, (F) 24 h ambulatory activity, (G) 24 h energy expenditure, (H) 24 h energy intake, (I-N) serum hormones and metabolites. (O-Q) Markers of brown and beige adipose tissue in BAT, PVAT and IWAT, $(\mathbf{R}-\mathbf{W})$ select metabolic genes in BAT and PVAT. Data expressed as mean $\pm \mathrm{SEM}, \mathrm{n}=4-5$ per group. For comparison, data was analysed by either one (A-E, $\mathbf{H}-\mathbf{Q})$, two-way ANOVA (F, R-W) or ANCOVA (G and H) with Sidak post hoc tests. Significance denoted as * <0.05; ** $<0.01$ or $^{* * *}<0.001$. 
Neither cold exposure, nor YM-178 were effective at inducing thermogenic genes (i.e., UCP1) in BAT or PVAT (Figure 2O-P) suggesting that chronic suppression of BAT from weaning reduces the ability of these thermogenic tissues to respond to a cold stimulus. Expression of the putative BAT and beige markers CITED1, and TMEM26, were reduced in BAT of cold exposed, and YM-178 treated rats, whilst the beige marker P2RX5 was upregulated in PVAT. Using targeted arrays to screen for primary genes involved in adipose tissue metabolism we saw an increase in the expression of FASN mRNA in both BAT and PVAT suggesting increased lipogenesis in both depots (Figure 2O,P). There was also an increase in genes involved in glycolysis (i.e., HK2 and PDK), fatty acid oxidation (i.e., ACACA and ACACB) and insulin resistance (i.e., AdipoR1 and MAPK9) in PVAT only following cold exposure (Figure 2R-W), demonstrating that this tissue is more responsive to cold than classic BAT when animals are raised at thermoneutrality. In contrast, in IWAT, UCP1 mRNA (Figure 2Q) was absent in all rats and, despite a c.125\% increase in IWAT mass of cold exposed rats, there was no change in the expression of other genes associated with thermogenesis (i.e., ADR 33 ), beige adipocytes (i.e., TMEM26) or lipogenesis (i.e., FASN) with this tissue being relatively unresponsive to all challenges.

Morphologically, BAT was characterised by a heterogenous mix of small, mitochondria rich lipid droplets and large lipid droplets, and adipocytes, indicative of large-scale whitening with thermoneutral housing from weaning (Figure 3A). Analysis of lipid droplet areas in BAT demonstrated a significant increase in both cold, and YM-178 treated rats (Figure 3B) with adipocyte size also increased in IWAT of cold-exposed rats (Figure 4A and B). Given the surprising increase in BAT and IWAT mass with cold exposure, we then carried out exploratory adipose tissue proteomics. This method, which quantifies the 30$40 \%$ most abundant proteins across samples, did not detect UCP1 in BAT, which is not unexpected given the chronic suppression of adaptive thermogenesis. We identified 175 differentially regulated proteins in BAT of cold-exposed rats (Figure 3C, Table 1 and Supplementary Data) including an increase in the mitochondrial citrate transporter protein (SLC25a1), glucose-6-phosphate dehydrogenase (G6PD), and the muscle isoforms of phosphoglycerate mutase (PGAM2) and creatine kinase (CKm).
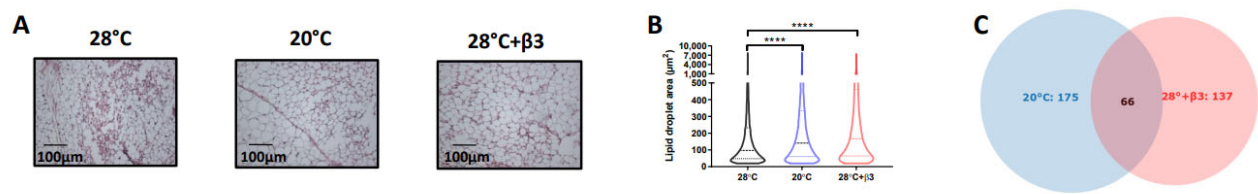

Reactome pathway and network analysis in BAT following cold-exposure
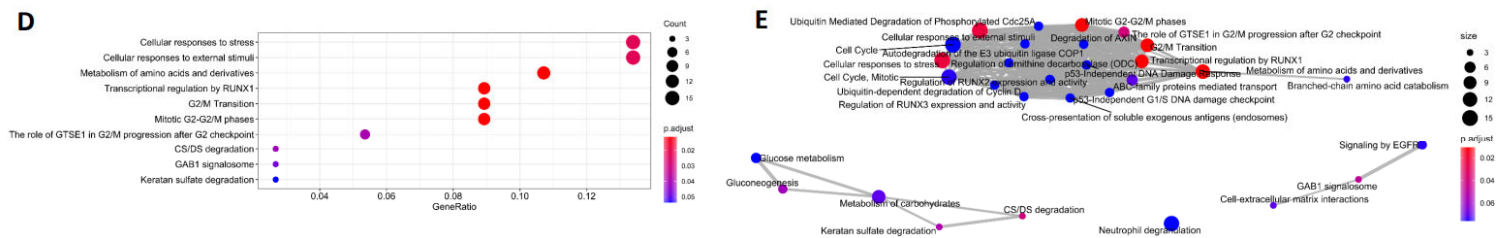

Reactome pathway and network analysis in BAT following Mirabegron
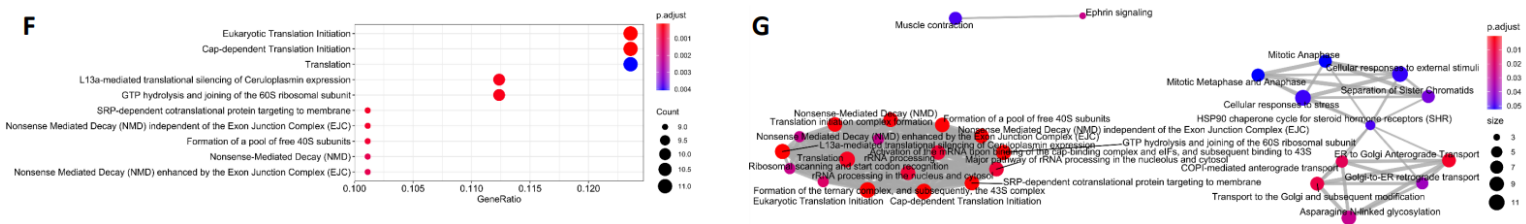

Figure 3. Histological and proteomics analysis of BAT following cold exposure $\left(20^{\circ} \mathrm{C}\right)$ and YM-178 treatment $\left(28^{\circ} \mathrm{C}+\beta 3\right)$. $(\mathbf{A}, \mathbf{B})$ Histological analysis of BAT and lipid droplet area. $(\mathbf{C})$ Venn diagram of differentially regulated proteins. Reactome pathway analysis detailing enriched pathways and 
interrelated networks in cold exposed $\left(20^{\circ} \mathrm{C}, \mathrm{D}, \mathbf{E}\right)$ and $\mathrm{YM}-178$ treated animals $\left(28^{\circ} \mathrm{C}+\beta 3, \mathbf{F}, \mathrm{G}\right)$. Data expressed as mean $\pm \mathrm{SEM}, \mathrm{n}=4-5$ per group. Adipocyte/lipid droplet area quantified using Adiposoft $\left(28^{\circ} \mathrm{C}, \mathrm{n}=8949 ; 20^{\circ} \mathrm{C}, 15512\right.$ and $\left.28^{\circ} \mathrm{C}+\beta 3,12446\right)$. For comparison, data was analysed by one-way ANOVA (B) or using the ReactomePA package (D-G). Significance denoted as ${ }^{* * *}<$ 0.0001 .
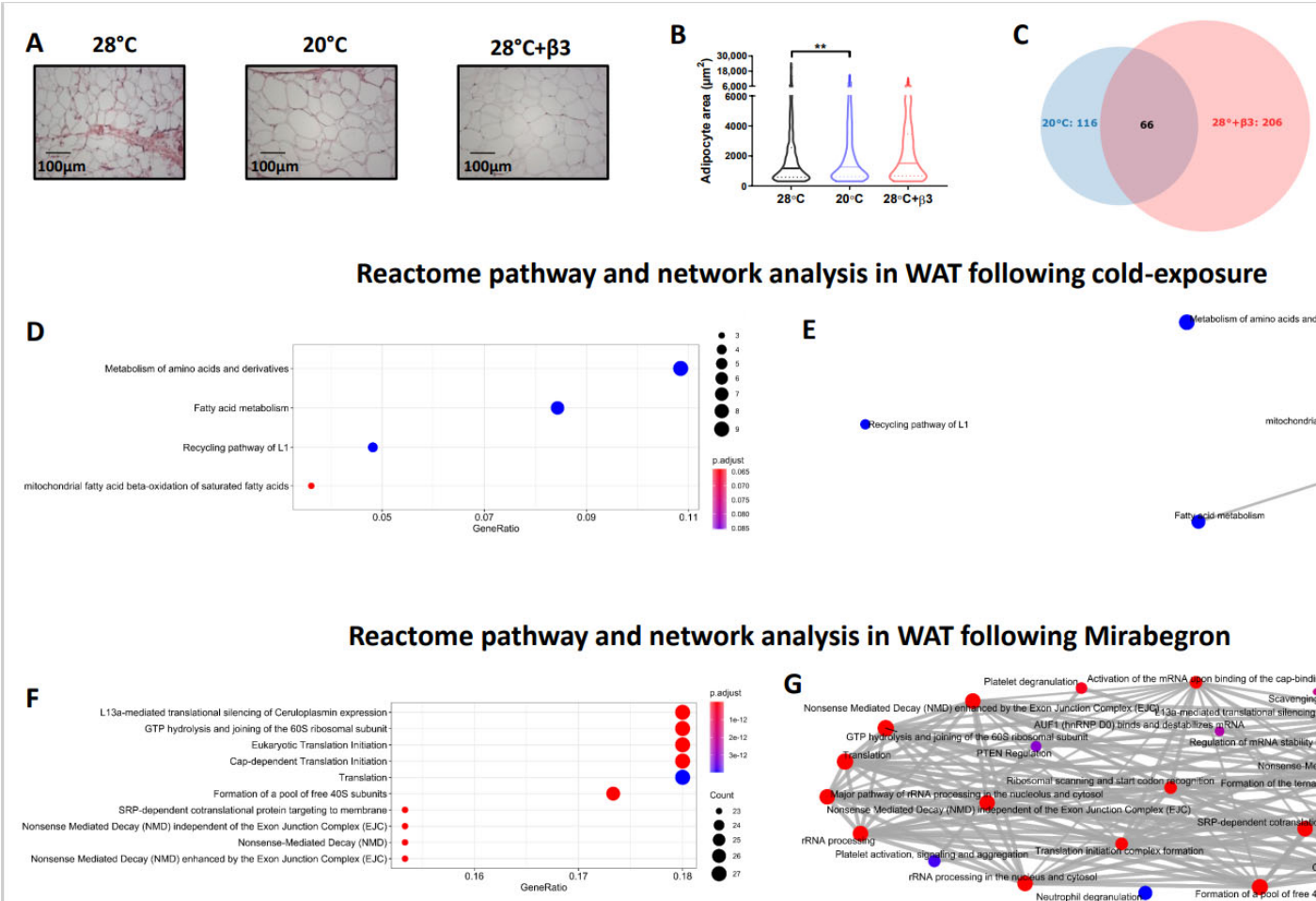

Reactome pathway and network analysis in WAT following cold-exposure

E
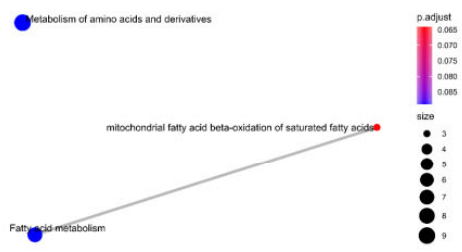

Figure 4. Histological and proteomics analysis of WAT following cold exposure $\left(20^{\circ} \mathrm{C}\right)$ and YM-178 treatment $\left(28^{\circ} \mathrm{C}+\beta 3\right)$. (A,B) Histological analysis of BAT and lipid droplet area. (C) Venn diagram of differentially regulated proteins. Reactome pathway analysis detailing enriched pathways and interrelated networks in cold-exposed $\left(20^{\circ} \mathrm{C}, \mathrm{D}, \mathrm{E}\right)$ and $\mathrm{YM}-178$ treated animals $\left(28^{\circ} \mathrm{C}+\beta 3, \mathrm{~F}, \mathrm{G}\right)$. Data expressed as mean $\pm \mathrm{SEM}, \mathrm{n}=4-5$ per group. Adipocyte/lipid droplet area quantified using Adiposoft $\left(28^{\circ} \mathrm{C}, \mathrm{n}=750 ; 20^{\circ} \mathrm{C}, 553\right.$ and $\left.28^{\circ} \mathrm{C}+\beta 3,527\right)$. For comparison, data was analysed by one way ANOVA (B) or using the ReactomePA package (D-G). Significance denoted as ${ }^{* *}<0.01$.

Table 1. Full list of differentially regulated proteins in brown adipose tissue.

\begin{tabular}{ccccc}
\hline Gene ID & Gene Name & Logfc & Adjpv \\
\hline $\mathbf{2 0}{ }^{\circ} \mathbf{C}$ & & & \\
80754 & Rabep2 & 3.69 & $6.35 \times 10^{-5}$ \\
114122 & Vcan & 2.74 & 0.000269 \\
292073 & Galns & -2.75 & 0.00027 \\
64012 & Rad50 & 1.50 & 0.000288 \\
171139 & Timm9 & -1.94 & 0.00037 \\
295088 & Gmps & 0.67 & 0.000396 \\
81716 & Ggcx & 1.44 & 0.000639 \\
25622 & Ptpn11 & -2.47 & 0.000975 \\
289590 & Ociad1 & -1.41 & 0.000988 \\
29384 & H2afy & -1.15 & 0.001077 \\
$\mathbf{2 8}{ }^{\circ} \mathbf{C}+\mathbf{B 3}$ & & & \\
116689 & Ptpn6 & -3.56 & 0.000154 \\
25650 & Atp1b1 & -1.40 & 0.000303 \\
\hline
\end{tabular}




\begin{tabular}{cccc}
59108 & Mb & 2.71 & 0.000548 \\
306262 & Btd & 2.72 & 0.000735 \\
501167 & Gmppa & 1.58 & 0.000934 \\
64528 & Golga2 & 0.98 & 0.001723 \\
287633 & Lrrc59 & -0.62 & 0.002902 \\
81726 & Mvd & -3.82 & 0.004012 \\
363425 & Cav2 & -2.41 & 0.004547 \\
114559 & Arhgef7 & -1.54 & 0.005187 \\
\hline
\end{tabular}

Conversely, 137 proteins were differentially regulated in BAT of YM-178 treated animals (Figure 3C, Table 1 and Supplementary Data) with an upregulation of proteins involved in skeletal muscle physiology including myosin heavy chain 4 (MYH4), fast-twitch skeletal muscle isoforms troponin I2 (TNNI2) and calsequestrin 1 (CASQ1) in addition to proteins governing endothelial adhesion and vascular growth (PECAM1 and FBLN5). These changes occurred alongside a downregulation of the aldo-keto reductase family member proteins B15 (AKR1B15) and C3 (AKR1C3), mevalonate diphosphate decarboxylase (MVD), trans-2,3-enoyl-CoA reductase (TECR), acyl-CoA dehydrogenase short/branched chain (ACADSB), phosphate cytidylyltransferase 1, choline, alpha (PCYT1A) and 3-hydroxybutyrate dehydrogenase 1 (BDH1) proteins.

We then carried out functional analysis of the BAT proteome. The differentially regulated proteins in BAT of cold-exposed animals enriched GO terms involved 'glucose import', 'ATP-dependent helicase activity' and 'regulation of protein phosphorylation' whilst there was also an enrichment of nuclear related GO terms including 'histone deacetylation', 'nucleosomal DNA binding', 'nuclear chromatin' and the 'nucleosome' (Table 2 and Supplementary Data). Conversely, differentially regulated proteins in BAT of animals treated with YM-178 enriched GO terms including 'positive regulation of protein kinase B signalling', 'negative regulation of cellular carbohydrate metabolic process' and 'positive regulation of atpase activity' whilst there was also an enrichment of GO terms involved in both brown adipocyte and muscle biology including 'brown fat cell differentiation', 'skeletal muscle contraction' and 'regulation of muscle contraction' (Table 2 and Supplementary Data). Finally, using ReactomePA we show enriched pathways regulated by cold exposure (Figure 3D), and their interactions (Figure 3E), including 'transcriptional regulation of RUNx1', pathways involved in mitosis (i.e., G2/M transition), and the degradation of glycoproteins (i.e., CS/DS degradation). Conversely, YM-178 treatment enriched multiple pathways associated with proteolysis (i.e., eukaryotic translation initiation and formation of a pool of free $40 \mathrm{~S}$ subunits) and RNA surveillance (i.e., nonsense mediated decay) (Figure 3F,G).

Table 2. Full list of GO terms enriched in brown adipose tissue.

\begin{tabular}{|c|c|c|c|c|}
\hline \multirow{2}{*}{$\frac{\text { Go ID }}{20^{\circ} \mathrm{C}}$} & \multirow[t]{2}{*}{ Go Name } & \multicolumn{3}{|c|}{ Count DE Count All Pv_Elim } \\
\hline & & & & \\
\hline \multicolumn{5}{|c|}{ Biological Process } \\
\hline GO:0000122 & $\begin{array}{l}\text { negative regulation of transcription from } \\
\text { RNA polymerase II promoter }\end{array}$ & 14 & 40 & 0.008 \\
\hline GO:0003006 & $\begin{array}{l}\text { developmental process involved in repro- } \\
\text { duction }\end{array}$ & 20 & 68 & 0.0146 \\
\hline GO:0071786 & $\begin{array}{l}\text { endoplasmic reticulum tubular network } \\
\text { organization }\end{array}$ & 3 & 4 & 0.0209 \\
\hline GO:0019098 & reproductive behavior & 3 & 4 & 0.0209 \\
\hline GO:0006544 & glycine metabolic process & 3 & 4 & 0.0209 \\
\hline GO:0016226 & iron-sulfur cluster assembly & 3 & 4 & 0.0209 \\
\hline GO:0090068 & positive regulation of cell cycle process & 7 & 17 & 0.0231 \\
\hline GO:0046323 & glucose import & 6 & 14 & 0.0285 \\
\hline
\end{tabular}


$\begin{array}{ll}\text { GO:0001932 } & \text { regulation of protein phosphorylation } \\ \text { neurotransmitter receptor transport to } \\ \text { postsynaptic membrane }\end{array}$

Molecular Function

GO:0000980 RNA polymerase II distal enhancer se-

GO:0030984 kininogen binding

GO:0031492 nucleosomal DNA binding

GO:0001846 opsonin binding

GO:0005212 structural constituent of eye lens

GO:0016634 oxidoreductase activity, acting on the $\mathrm{CH}$ $\mathrm{CH}$ group of donors, oxygen as acceptor

GO:0016831 carboxy-lyase activity

GO:0016746 transferase activity, transferring acyl groups

GO:0004616 phosphogluconate dehydrogenase (decarboxylating) activity

GO:0008484 sulfuric ester hydrolase activity

\section{Cellular Component}

GO:0042582 azurophil granule

GO:0000790 nuclear chromatin

GO:0031616 spindle pole centrosome

GO:0000786 nucleosome

GO:0042719 mitochondrial intermembrane space protein transporter complex

GO:0001740 Barr body

GO:0072687 meiotic spindle

GO:0034751 aryl hydrocarbon receptor complex

GO:0043196 varicosity

GO:0001931 uropod

$28^{\circ} \mathrm{C}+\mathrm{B} 3$

Biological Process

GO:0003009 skeletal muscle contraction

GO:0051897 positive regulation of protein kinase B signaling

GO:0010677 negative regulation of cellular carbohydrate metabolic process

GO:1901896 positive regulation of calcium-transporting ATPase activity

GO:0032781 positive regulation of ATPase activity

GO:0050873 brown fat cell differentiation

GO:0006937 regulation of muscle contraction

GO:0071560 cellular response to transforming growth factor beta stimulus

GO:0060048 cardiac muscle contraction

GO:0048193 Golgi vesicle transport

\section{Molecular Function}

GO:0035259 glucocorticoid receptor binding

GO:0001671 ATPase activator activity

GO:0008134 transcription factor binding

GO:0050431 transforming growth factor beta binding
129

0.0316

2

0.0333

4

3

4

3

\section{5}

0.0046

$3 \quad 0.006$

$\begin{array}{ll}6 & 0.0119\end{array}$

$\begin{array}{ll}4 & 0.0207\end{array}$

$\begin{array}{ll}4 & 0.0207\end{array}$

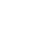

\section{4}

0.0207

$\begin{array}{ll}10 & 0.022\end{array}$

9

0.0331

0.0331

0.0011

0.0014

0.0209

0.0223

0.0333

0.0333

0.0333

0.0333

0.0333

0.0453

$5 \quad 0.0453$

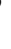




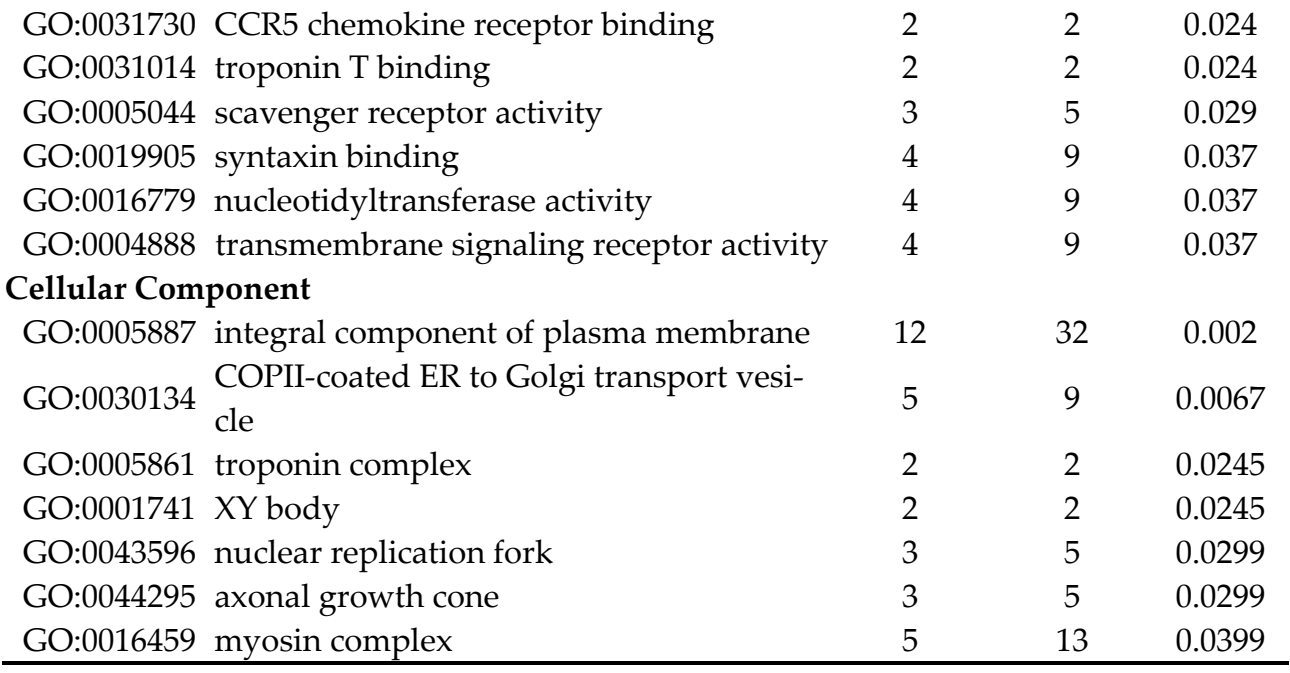

The substantial increase in IWAT of cold-exposed rats was associated with 116 differentially regulated proteins (Figure 4C, Table 3 and Supplementary Data) including the upregulation of lipid metabolic proteins such as fatty acid binding proteins 1 (FABP1) and 3 (FABP3), fatty acid synthase (FASN) and ATP citrate lyase (ACLY) and those involved in beta oxidation, including hydroxynacyl-CoA dehydrogenase (HADH) and acyl-CoA dehydrogenase long chain (ACADL). In addition, there was an upregulation of glycerol kinase (GK), pyruvate carboxylase (PC) and monocarboxylic acid transporter 1 (MCT1) that were accompanied by a downregulation of multiple proteins involved in mRNA processing and splicing (i.e., RNA binding motif protein 8B, RBM8B; dexd-Box helicase 39B, DDX39B and poly(u) binding splicing factor 60, PUF60).

Table 3. Full list of differentially regulated proteins in white adipose tissue.

\begin{tabular}{ccccc}
\hline Gene ID & Gene Name & Logfc & Adjpv \\
\hline $\mathbf{2 0}^{\circ} \mathbf{C}$ & & & \\
& & & -2.79 & $8.97 \times 10^{-6}$ \\
& Bin1 & -2.68 & $4.47 \times 10^{-5}$ \\
304290 & Kdelr2 & -2.65 & 0.000108 \\
24667 & Ppm1b & -1.35 & 0.000125 \\
84114 & Agps & -2.94 & 0.000753 \\
84401 & Puf60 & 0.94 & 0.000791 \\
300983 & Abhd14b & -2.40 & 0.000854 \\
29218 & Rcn2 & -0.94 & 0.002064 \\
290028 & Osgep & -2.34 & 0.00248 \\
24230 & Tspo & -2.15 & 0.00629 \\
171452 & Rab3il1 & & \\
$\mathbf{2 8}{ }^{\circ} \mathbf{C}+\mathbf{B 3}$ & & -3.75 & $6.93 \times 10^{-5}$ \\
83730 & Vamp8 & 1.51 & 0.000111 \\
29521 & Scamp1 & 0.92 & 0.000382 \\
25116 & Hsd11b1 & -0.69 & 0.000942 \\
117045 & Eif4e & 1.79 & 0.001104 \\
25342 & Oxtr & 0.85 & 0.001124 \\
298566 & C1qa & -0.65 & 0.001133 \\
445268 & Ufc1 & 0.61 & 0.00204 \\
78947 & Gcs1 & 0.87 & 0.004234 \\
266734 & Npas4 & 0.78 & 0.004516 \\
\hline 246303 & Serbp1 &
\end{tabular}


YM-178 administration modulated 206 proteins in IWAT (Figure 4C, Table 3 and Supplementary Data) including an upregulation of multiple proteins involved in the nervous system including synuclein gamma (SNCG), neurolysin (NLN) and neuronal pas domain protein 4 (NPAS4). This was associated with an increase in proteins involved in lipid and cholesterol metabolism including carnitine palmitoyltransferase 1A (CPT1A), hormone-sensitive lipase (LIPE) and apolipoprotein $\mathrm{C} 1$ and $\mathrm{M}$ (APOC1 and APOM). Interestingly, YM-178 also induced an increase in multiple inflammatory proteins, including orosomucoid 1 (ORM1), complement C4A (C4A), S100 calcium binding protein A8 (S100A8) and S100 calcium binding protein B (S100B).

Differentially regulated proteins in IWAT of cold-exposed rats enriched GO terms involved in the 'DNA damage response', '3-hydroxyacyl-coa dehydrogenase activity' and 'NAD+ binding' whilst YM-178 enriched inflammatory terms including the 'acute phase response' and 'structural constituent of ribosome' suggesting an effect of sympathetic activation on both the inflammatory system and protein synthesis (Table 4 and Supplementary Data). Finally, ReactomePA demonstrated enriched pathways (Figure 4D), and their interactions (Figure 4E) were associated with IWAT expansion including the 'metabolism of amino acids and derivatives' and 'fatty acid metabolism'. Conversely, and similar to BAT, YM-178 treatment enriched multiple pathways associated with proteolysis (i.e., eukaryotic translation initiation and formation of a pool of free 40S subunits) and RNA surveillance (i.e., nonsense mediated decay) but to a larger degree (i.e., 23-27 proteins per term rather than 9-11; Figure $4 \mathrm{~F}, \mathrm{G}$ ).

Table 4. Full list of GO terms enriched in white adipose tissue.

\begin{tabular}{|c|c|c|c|c|}
\hline Go ID & Go Name & Count DE & Count All & Pv_Elim \\
\hline \multicolumn{5}{|c|}{$20^{\circ} \mathrm{C}$} \\
\hline \multicolumn{5}{|c|}{ Biological Process } \\
\hline GO:0030330 & $\begin{array}{l}\text { DNA damage response, signal } \\
\text { transduction by p53 class mediator }\end{array}$ & 5 & 6 & 0.0022 \\
\hline GO:0048711 & $\begin{array}{l}\text { positive regulation of astrocyte dif- } \\
\text { ferentiation }\end{array}$ & 3 & 3 & 0.0023 \\
\hline GO:0071498 & $\begin{array}{l}\text { cellular response to fluid shear } \\
\text { stress }\end{array}$ & 3 & 3 & 0.0023 \\
\hline GO:0032780 & $\begin{array}{l}\text { negative regulation of ATPase ac- } \\
\text { tivity }\end{array}$ & 3 & 3 & 0.0023 \\
\hline GO:0051607 & defense response to virus & 5 & 9 & 0.003 \\
\hline GO:0001822 & kidney development & 11 & 35 & 0.0036 \\
\hline GO:0050731 & $\begin{array}{l}\text { positive regulation of peptidyl-ty- } \\
\text { rosine phosphorylation }\end{array}$ & 7 & 17 & 0.0037 \\
\hline GO:0002244 & $\begin{array}{l}\text { hematopoietic progenitor cell dif- } \\
\text { ferentiation }\end{array}$ & 3 & 4 & 0.0081 \\
\hline GO:0045577 & regulation of B cell differentiation & 3 & 4 & 0.0081 \\
\hline GO:0042130 & $\begin{array}{l}\text { negative regulation of } \mathrm{T} \text { cell prolif- } \\
\text { eration }\end{array}$ & 3 & 4 & 0.0081 \\
\hline \multicolumn{5}{|c|}{ Molecular Function } \\
\hline GO:0051287 & NAD binding & 11 & 31 & 0.0013 \\
\hline GO:0008144 & drug binding & 9 & 29 & 0.0101 \\
\hline GO:0005001 & $\begin{array}{l}\text { transmembrane receptor protein } \\
\text { tyrosine phosphatase activity }\end{array}$ & 2 & 2 & 0.0178 \\
\hline GO:0005521 & lamin binding & 3 & 5 & 0.0191 \\
\hline GO:0042393 & histone binding & 5 & 13 & 0.021 \\
\hline GO:0033613 & $\begin{array}{l}\text { activating transcription factor } \\
\text { binding }\end{array}$ & 3 & 6 & 0.0345 \\
\hline
\end{tabular}




\begin{tabular}{|c|c|c|c|c|}
\hline GO:0003857 & $\begin{array}{l}\text { 3-hydroxyacyl-CoA dehydrogen- } \\
\text { ase activity }\end{array}$ & 3 & 6 & 0.0345 \\
\hline GO:0045296 & cadherin binding & 22 & 113 & 0.0365 \\
\hline GO:0004028 & $\begin{array}{l}\text { 3-chloroallyl aldehyde dehydro- } \\
\text { genase activity }\end{array}$ & 2 & 3 & 0.0486 \\
\hline GO:0071933 & Arp2/3 complex binding & 2 & 3 & 0.0486 \\
\hline \multicolumn{5}{|c|}{ Cellular Component } \\
\hline GO:0005884 & actin filament & 9 & 24 & 0.0021 \\
\hline GO:0032993 & protein-DNA complex & 5 & 11 & 0.0088 \\
\hline GO:0002102 & podosome & 7 & 14 & 0.013 \\
\hline GO:0016607 & nuclear speck & 8 & 26 & 0.0146 \\
\hline GO:0031209 & SCAR complex & 2 & 2 & 0.0172 \\
\hline GO:0042611 & MHC protein complex & 2 & 2 & 0.0172 \\
\hline GO:0005687 & U4 snRNP & 2 & 2 & 0.0172 \\
\hline GO:0005856 & cytoskeleton & 49 & 238 & 0.0284 \\
\hline GO:0030054 & cell junction & 41 & 216 & 0.0291 \\
\hline GO:0005681 & spliceosomal complex & 9 & 22 & 0.0311 \\
\hline \multicolumn{5}{|l|}{$28^{\circ} \mathrm{C}+\mathrm{B} 3$} \\
\hline \multicolumn{5}{|c|}{ Biological Process } \\
\hline GO:0006953 & acute-phase response & 7 & 12 & 0.0035 \\
\hline GO:0000381 & $\begin{array}{l}\text { regulation of alternative mRNA } \\
\text { splicing, via spliceosome }\end{array}$ & 7 & 12 & 0.0035 \\
\hline GO:0034113 & heterotypic cell-cell adhesion & 8 & 12 & 0.0061 \\
\hline GO:0070528 & protein kinase C signaling & 4 & 5 & 0.0063 \\
\hline GO:0015671 & oxygen transport & 4 & 5 & 0.0063 \\
\hline GO:1901741 & $\begin{array}{l}\text { positive regulation of myoblast fu- } \\
\text { sion }\end{array}$ & 3 & 3 & 0.0077 \\
\hline GO:0070934 & $\begin{array}{l}\text { CRD-mediated mRNA stabiliza- } \\
\text { tion }\end{array}$ & 3 & 3 & 0.0077 \\
\hline GO:0007566 & embryo implantation & 6 & 12 & 0.0179 \\
\hline GO:0040007 & growth & 29 & 103 & 0.0209 \\
\hline GO:0071345 & $\begin{array}{l}\text { cellular response to cytokine stim- } \\
\text { ulus }\end{array}$ & 25 & 86 & 0.0211 \\
\hline \multicolumn{5}{|c|}{ Molecular Function } \\
\hline GO:0003735 & structural constituent of ribosome & 24 & 62 & 0.00037 \\
\hline GO:0005344 & oxygen carrier activity & 4 & 5 & 0.00657 \\
\hline GO:0003730 & mRNA 3'-UTR binding & 8 & 18 & 0.01548 \\
\hline GO:0003682 & chromatin binding & 10 & 25 & 0.01623 \\
\hline GO:0140097 & catalytic activity, acting on DNA & 5 & 9 & 0.01908 \\
\hline GO:0042162 & telomeric DNA binding & 3 & 4 & 0.02688 \\
\hline GO:0045294 & alpha-catenin binding & 3 & 4 & 0.02688 \\
\hline GO:0004527 & exonuclease activity & 3 & 4 & 0.02688 \\
\hline GO:0003723 & RNA binding & 73 & 281 & 0.02734 \\
\hline GO:0019825 & oxygen binding & 4 & 7 & 0.03278 \\
\hline \multicolumn{5}{|c|}{ Cellular Component } \\
\hline GO:0022625 & cytosolic large ribosomal subunit & 15 & 30 & 0.00018 \\
\hline GO:0016323 & basolateral plasma membrane & 14 & 32 & 0.00164 \\
\hline GO:0005833 & hemoglobin complex & 3 & 3 & 0.00782 \\
\hline GO:0005903 & brush border & 10 & 23 & 0.00812 \\
\hline GO:0030864 & cortical actin cytoskeleton & 9 & 20 & 0.00918 \\
\hline GO:0016327 & apicolateral plasma membrane & 3 & 4 & 0.02666 \\
\hline
\end{tabular}




\begin{tabular}{llccc} 
GO:0044451 & nucleoplasm part & 18 & 58 & 0.0269 \\
GO:0005637 & nuclear inner membrane & 5 & 10 & 0.03167 \\
GO:0035770 & ribonucleoprotein granule & 11 & 32 & 0.03804 \\
GO:0097225 & sperm midpiece & 2 & 2 & 0.03953 \\
\hline
\end{tabular}

\section{Discussion}

Housing temperature, especially cold exposure, impacts on metabolic homeostasis as illustrated by the effects on BAT and 'browning' [3,15-18]. Little is known as to whether interventions considered to promote browning are effective in animals maintained at thermoneutrality $[2,18]$. Here we show that chronic exposure to a mild cold stimulus (i.e., standard housing temperature) drives weight gain and the deposition of large quantities of subcutaneous adipose tissue in obese rats rather than the activation of BAT and subsequent weight-loss as expected [1]. This effect is not seen in rats treated with the highly selective $\beta 3$-agonist YM-178, suggesting sympathetic activation is not involved and it is a direct effect of ambient temperature. Taken together these findings are indicative of a novel mechanism whereby rats increase body weight and fat mass following chronic suppression of adaptive thermogenesis from weaning.

There is accumulating evidence that, in the absence of adaptive thermogenesis, other mechanisms compensate in order to maintain body temperature. For instance, in UCP1 $\mathrm{k} / \mathrm{o}$ mice, a reduction in BAT thermogenesis leads to the recruitment of shivering thermogenesis in skeletal muscle [19]. Conversely, when shivering is impaired in Sarcolipin k/o mice, there is a compensatory increase in BAT activity [19]. In obese animals lacking BAT, there seems to be an entirely different homeostatic response to cold stress. Following BAT lipectomy, obese, cold-exposed rats gain weight and adipose tissue mass is nearly doubled [20] which mirrors our findings when BAT is chronically supressed. Furthermore, intermittent cold exposure (ICE, from $20^{\circ} \mathrm{C}$ to $4^{\circ} \mathrm{C}$ ) over a period of days promotes weight gain and adiposity and this is associated with intermittent increases in energy intake [21]. Rats are also more susceptible to weight gain, and fat accumulation when reared in the cold $\left(18{ }^{\circ} \mathrm{C}\right.$ vs. $\left.30^{\circ} \mathrm{C}\right)$, an effect which persists when housed at a common temperature [22]. More recently, it was demonstrated that ICE increases weight gain, largely due to increases in adiposity, in both male and female mice housed at thermoneutrality and fed a diet high in fat [5]. Despite no effect on circulating NEFA or insulin concentrations, ICE increased lipid deposition in hepatocytes and circulating leptin whilst decreasing circulating triglycerides. Intriguingly, whilst ICE exacerbated diet-induced obesity it let to marked improvements in glucose homeostasis. This suggests that in human relevant conditions, i.e., thermoneutrality, both chronic and intermittent cold exposure may exacerbate weight gain through as yet unidentified mechanisms, but may, in the case of ICE, improve glucose homeostasis.

Whilst recent work on ICE demonstrated that cold-induced weight gain was dependant on energy intake, we did not see increased food intake suggesting another, unexplained mechanism, whereby cold induces adiposity. There is a plausible mechanism linking the gut to this phenotype which needs to be explored in future. Cold exposure drives intestinal growth, increased fatty acid absorption and paracellular permeability to nutrients $[23,24]$. The efficiency of energy utilisation is also sufficient to maintain core body temperature during acute cold exposure [25]. If the gut can grow, even during periods of energy restriction, and maximise absorption of energy to a degree that it sustains critical functions, and ultimately life, then it may be able to drive adiposity in a similar manner. Whilst the insulative effects of obesity are being debated [26-28] a model whereby rats, and potentially other rodents, deposit subcutaneous fat during exposure to cold (i.e., "store up nuts for the winter internally" [22]) would make sense, and be hugely beneficial from an evolutionary perspective given wild animals cannot simply increase energy intake during winter months. 
Another important finding of this study is that the increase in weight gain and subcutaneous AT mass seen in cold-exposed rats was not associated with impaired metabolic parameters (i.e., fasting glucose and lipids) or any discernible adipose tissue dysfunction. Here, chronic exposure to a mild cold stimulus seemingly drives a phenotypically healthy expansion of subcutaneous AT. Using exploratory proteomics, we were able to elucidate processes in both BAT and IWAT associated with this expansion of AT mass. An increase in proteins that modulate metabolism, including those involved in glycolysis, the TCA cycle and lipogenesis, suggests that there is an increased metabolic flux in these depots which, ultimately, results in net lipogenesis. In BAT, this is associated with an enrichment of mitotic pathways (i.e., G2/M transition). The G2/M transition is a critical point in the cell cycle where, following DNA replication, the mitotic process begins, and cells separate into replicate daughter cells. Mitotic clonal expansion is essential for adipogenesis in 3T3L1 adipocytes [29] and is one of the postulated mechanisms through which FTO regulates fat mass [30]. Further, enrichment of the 'transcriptional regulation on RUNX1' pathway, which regulates the white-to-brown/beige transition via CDK6 points towards the cellcycle as a key mediator of increased BAT mass following cold exposure [31]. As such, an increase in mitosis of adipocyte pre-cursors, key changes in the cell cycle and subsequent adipogenesis may be partly driving increased BAT mass.

In IWAT, an upregulation of regulatory proteins involved in lipid metabolism (i.e., FABP1, FASN and ACLY) and beta oxidation (i.e., HADH and ACADL) in addition to GK, PC and MCT1 indicates an increased metabolic flux in IWAT. Importantly, however, adipocyte size in IWAT of cold-exposed rats only increased by $\sim 17 \%$. As such, we predict that this physiological expansion of IWAT is largely due to adipogenesis which ties in to the already established theory of 'healthy adipose tissue expansion' where growth of subcutaneous AT, through adipogenesis, protects from the adverse effects of an obesogenic diet [32].

We were also able to elucidate the impact of sympathetic activation in the absence of any change in housing temperature by administering YM-178. This highly selective $\beta 3-$ agonist increases BAT activity, whole body EE and induces 'browning' in humans [3336]. YM-178 has a 20-200 higher agonist activity for both rat, and human bladder than other agonists and, despite differences in $\beta$-AR subtype expression between rats and humans, exhibits full agonistic activity in both species [37-40]. Recently, two landmark studies have demonstrated that YM-178 also improves glucose homeostasis and insulin sensitivity, improves $\beta$-cell function, reduces skeletal muscle triglyceride content and increases HDL cholesterol and adiponectin in overweight and obese prediabetic or insulin resistant humans [10,11]. However, these, and other studies show that human BAT activity and the response to either cold, or other thermogenic stimuli (i.e., YM-178) is highly heterogenous which is unsurprising given humans undergo seasonal BAT activation to varying degrees [41]. Our aim was to determine the efficacy of this treatment in a homogenous population where BAT had been supressed from early life. Under these conditions, YM-178 treatment had no impact on metabolic parameters or thermogenesis. It does, however, regulate pathways involved in protein synthesis in both BAT and, to a larger degree, IWAT. In the hippocampus, $\beta 3$-AR agonism (by isoprenaline) drives ERK/MTOR dependent activation of eukaryotic initiation factor $4 \mathrm{E}$ and inhibition of the translation repressor 4E-BP whilst CL316, 243 and salbutamol drive skeletal muscle hypertrophy and protein synthesis in mice and humans, respectively [42-44]. This would suggest a novel role for YM-178 in regulating protein synthesis in adipose tissues which has yet to be examined. YM-178 also regulated proteins involved in the acute phase response and inflammation in both BAT and IWAT and increased lipid droplet size in BAT. It is unclear why, or how, $\beta 3$-AR agonism would increase lipid droplet size in this setting however, when taken together, this suggests YM-178 treatment following chronic suppression of BAT may act negatively on AT driving inflammatory processes and lipid droplet/adipocyte hypertrophy. Whilst these differences may be species specific, they may also highlight potential 
effects of YM-178 if given to individuals lacking BAT, or who exhibit low responsiveness to thermogenic stimuli.

Whilst this study points to a novel, unexplained yet controversial adaptation to cold, it is important to remember the major differences in physiology and cellular processes in animals housed at different ambient temperatures [18]. These differences are likely exacerbated in animals who have been raised at thermoneutrality from weaning and it is not entirely unexpected that the physiological response to a commonly studied stimulus would be different in this scenario. It is clear that the early life environment, and early life stressors, impact adult physiology and susceptibility to disease, including obesity, and important adaptations during the developmental period may underlie the adaptations seen here. Despite aiming to mimic human physiology, this work cannot replicate the human in-utero, and birth environments, and there may be other key developmental changes in humans during this period that are not applicable here. Further, rearing temperature of rats is associated with changes in sympathoadrenal activity and rats are susceptible to obesity in a strain- and sex-dependent manner [22,45-47]. It will be important in future to determine if this effect is not only species specific, but also strain and sex specific as we look to untangle aspects of this biology to promote healthy expansion of adipose tissue in humans. Finally, whilst many of our OMICs hits are regulated by, or critical for the adaptation to cold or $\beta 3$-AR agonism at standard housing temperatures this dataset provides a rich resource for further analysis of novel proteins associated with weight gain.

\section{Conclusions}

In summary, we show that chronic exposure to mild cold following chronic suppression of BAT drives weight gain and the deposition of large quantities of subcutaneous AT. Whilst the precise mechanism is not clear, this work points towards a novel response whereby animals increase body weight and fat mass in response to a reduction in ambient temperature. We propose that chronic suppression of BAT from weaning, using thermoneutral housing and an obesogenic diet, changes the physiological response to cold in the obese state. Instead, humanised animals deposit adipose tissue and gain weight, an effect not seen with YM-178, suggesting a direct effect of temperature, where an insulative mechanism is potentially recruited when a thermogenic response is absent.

\section{Methods}

\subsection{Animals, Cold Exposure and YM-178 Treatment}

All studies were approved by the University of Nottingham Animal Welfare and Ethical Review Board and were carried out in accordance with the UK Animals (Scientific Procedures) Act of 1986 (PPL no.). Eighteen male Sprague-Dawley rats aged 3 weeks were obtained from Charles River (Kent, UK) and housed immediately at thermoneutrality (c. $28^{\circ} \mathrm{C}$ ), on a high-fat diet (HFD; $45 \%, 824,018$ SDS, Kent, UK), under a 12:12-h reverse lightdark cycle (lights off at 08:00). These conditions were chosen to closer mimic human physiology [3], minimise animal stress and maximise data quality and translatability [48]. At 12 weeks of age, all animals were randomised to either 4 weeks of standard housing temperature $\left(20^{\circ} \mathrm{C}, \mathrm{n}=6\right)$, daily $\mathrm{YM}-178$ administration by oral gavage $\left(28^{\circ} \mathrm{C}+\beta 3\right.$, at a clinically relevant dose of $0.75 \mathrm{mg} / \mathrm{kg} / \mathrm{day}, \mathrm{n}=6)$ or HFD controls $\left(28^{\circ} \mathrm{C}, \mathrm{n}=6\right)$. Animals randomised to $20^{\circ} \mathrm{C}$ were moved into an adjacent, climate-controlled room, for the 4-week period. In adherence to the National Centre for the Replacement, Refinement and Reduction of Animals in Research (NC3Rs), this experiment was run alongside our work looking at the effect of exercise training on 'browning' and utilised the same cohort of control HFD animals [2].

\subsection{Metabolic Assessment and Tissue Collection}

All animals were placed in an open-circuit calorimeter (CLAMS: Columbus Instruments, Linton Instrumentation, Palgrave, Norfolk, UK) for the final $48 \mathrm{~h}$ to enable the 
assessment of whole-body metabolism [49]. All animals were then weighed and fasted overnight prior to euthanasia by rising $\mathrm{CO}_{2}$ gradient. BAT, perivascular BAT (PVAT) from the thoracic aorta and IWAT were then rapidly dissected, weighed, snap-frozen in liquid nitrogen and stored at $-80{ }^{\circ} \mathrm{C}$ for subsequent analysis.

\subsection{Histology}

Adipose tissue histology was performed as previously described [8]. Briefly, BAT and IWAT were fixed in formalin, embedded in wax using an Excelsior ES processor (Thermo-Fisher, Runcorn, Cheshire, UK) and stained using haematoxylin and eosin (Sigma-Aldrich, Gillingham, Dorset, UK). Between 3 and 5 random sections were then imaged at 10x with an Olympus BX40 microscope and adipocyte size was quantified using Adiposoft [50].

\subsection{Gene Expression Analysis}

Total RNA was extracted from each fat depot using the RNeasy Plus Micro extraction kit (Qiagen, West Sussex, UK) using an adapted version of the single step acidified phenol-chloroform method. RT-qPCR was carried out as previously described using rat-specific oligonucleotide primers (Sigma) or FAM-MGB Taqman probes [49]. Gene expression was determined using the GeNorm algorithm against two selected reference genes; RPL19:RPL13a in BAT and IWAT (stability value $\mathrm{M}=0.163$ in BAT and 0.383 in IWAT) and RPL19:HPRT1 in PVAT (stability value $\mathrm{M}=0.285$ ).

\subsection{Serum Analysis}

Glucose (GAGO-20, Sigma-Aldrich, Gillingham, UK), triglycerides (LabAssay Trigylceride, Wako, Neuss, Germany), non-esterified fatty acids (NEFA-HR(2), Wako, Neuss, Germany), insulin (80-INSRT-E01, Alpco, Salem, NH, USA) and leptin (EZRL-83K, Merck, Darmstadt, Germany) were measured as previously described following the manufacturer's instructions [8].

\subsection{Adipose Tissue Proteomics}

Protein extraction, clean up and trypsinisation was performed on 50-100 mg of frozen tissue ( $\mathrm{n}=4$ /group) was homogenised in $500 \mu \mathrm{L}$ CellLytic MT cell lysis buffer (Sigma, C3228) prior to removal of lipid and other contaminants using the ReadyPrep 2D cleanup Kit (Biorad, 1632130) [49]. Samples were then subjected to reduction, alkylation and overnight trypsinisation following which they were dried down at $60^{\circ} \mathrm{C}$ for $4 \mathrm{~h}$ and stored at $80{ }^{\circ} \mathrm{C}$ before resuspension in LCMS grade $5 \%$ acetonitrile in $0.1 \%$ formic acid for subsequent analysis. Analysis by mass spectrometry was carried out on a SCIEX TripleTOF 6600 instrument [51] with samples analysed in both SWATH (Data Independent Acquisition) and IDA (Information Dependent Acquisition) modes for quantitation and spectral library generation respectively. IDA data was searched together using ProteinPilot 5.0.2 to generate a spectral library and SWATH data was analysed using Sciex OneOmics software [52] extracted against the locally generated library as described previously [49].

\subsection{Statistical Analysis}

Statistical analysis was performed in GraphPad Prism version 8.0 (GraphPad Software, San Diego, CA, USA). Data are expressed as mean \pm SEM with details of specific statistical tests in figure legends. Despite prior use of the control group to understand the impact of exercise training, only the groups included in this paper were utilised for analyses [8]. Functional analysis of the proteome was performed using the Advaita Bioinformatic iPathwayGuide software (www.advaitabio.com/ipathwayguide.html) (fold change \pm 0.5 and confidence score cut-off of 0.75 ). Significantly impacted biological processes and molecular functions were analysed in the context of the Gene Ontology Consortium database (November 2017) [53]. The Elim pruning method, which removes genes mapped to 
a significant GO term from more general (higher level) GO terms, was used to overcome the limitation of errors introduced by considering genes multiple times [54]. Pathway analysis was carried out using the ReactomePA package on R Studio (version 3.6.2) with a false discovery rate of $<0.1$.

Supplementary Materials: The following supporting information can be downloaded at: www.mdpi.com/article/10.3390/ijms23031869/s1.

Author Contributions: P.A., H.B. and M.E.S. conceived the study and attained the funding; P.A. and M.E.S. developed and designed the experiments; P.A., J.E.L., I.L., A.K.M., I.B., R.C. and D.J.B. performed the experiments; P.A., A.K.M. and D.J.B. analysed the data; P.A. and M.E.S. wrote the paper which was revised critically by D.J.B., H.B., F.J.P.E. and J.E.L. for important intellectual content. All authors have read and agreed to the published version of the manuscript.

Funding: This research was funded by The British Heart Foundation, grant number FS/15/4/31184/] and the APC was funded by the University of Nottingham/British Heart Foundation

Institutional Review Board Statement: All studies were approved by the University of Nottingham Animal Welfare and Ethical Review Board (PPL 403560), and were carried out in accordance with the UK Animals (Scientific Procedures) Act of 1986.

Informed Consent Statement: Not applicable.

Data Availability Statement: The datasets used and analysed during the current study are available from the corresponding author on reasonable request.

Conflicts of Interest: The authors declare no conflict of interest.

\section{References}

1. Chechi, K.; van Marken Lichtenbelt, W.; Richard, D. Brown and beige adipose tissues: Phenotype and metabolic potential in mice and men. J. Appl. Physiol. 2018, 124, 482-496. https://doi.org/10.1152/japplphysiol.00021.2017.

2. Gordon, C.J. The mouse thermoregulatory system: Its impact on translating biomedical data to humans. Physiol. Behav. 2017, 179, 55-66. https://doi.org/10.1016/j.physbeh.2017.05.026.

3. Kalinovich, A.V.; de Jong, J.M.; Cannon, B.; Nedergaard, J. UCP1 in adipose tissues: Two steps to full browning. Biochimie 2017, 134, 127-137. https://doi.org/10.1016/j.biochi.2017.01.007.

4. $\quad$ de Jong, J.M.A.; Sun, W.; Pires, N.D.; Frontini, A.; Balaz, M.; Jespersen, N.Z.; Feizi, A.; Petrovic, K.; Fischer, A.W.; Bokhari, M.H.; et al. Human brown adipose tissue is phenocopied by classical brown adipose tissue in physiologically humanized mice. Nat. Metab. 2019, 1, 830-843. https://doi.org/10.1038/s42255-019-0101-4.

5. McKie, G.L.; Shamshoum, H.; Hunt, K.L.; Thorpe, H.H.A.; Dibe, H.A.; Khokhar, J.Y.; Doucette, C.A.; Wright, D.C. Intermittent cold exposure improves glucose homeostasis despite exacerbating diet-induced obesity in mice housed at thermoneutrality. J. Physiol. 2021, https://doi.org/10.1113/JP281774.

6. Symonds, M.E.; Pope, M.; Budge, H. The Ontogeny of Brown Adipose Tissue. Annu. Rev. Nutr. 2015, 35, $295-320$.

7. Iannaccone, P.M.; Jacob, H.J. Rats! Dis. Model. Mech. 2009, 2, 206-210. https://doi.org/10.1242/dmm.002733.

8. Aldiss, P.; Lewis, J.E.; Lupini, I.; Bloor, I.; Chavoshinejad, R.; Boocock, D.J.; Miles, A.K.; Ebling, F.J.P.; Budge, H.; Symonds, M.E. Exercise Training in Obese Rats Does Not Induce Browning at Thermoneutrality and Induces a Muscle-Like Signature in Brown Adipose Tissue. Front. Endocrinol. 2020, 11, 97. https://doi.org/10.3389/fendo.2020.00097.

9. Tsiloulis, T.; Carey, A.L.; Bayliss, J.; Canny, B.; Meex, R.C.R.; Watt, M.J. No evidence of white adipocyte browning after endurance exercise training in obese men. Int. J. Obes. 2018, 42, 721-727. https://doi.org/10.1038/ijo.2017.295.

10. O'Mara, A.E.; Johnson, J.W.; Linderman, J.D.; Brychta, R.J.; McGehee, S.; Fletcher, L.A.; Fink, Y.A.; Kapuria, D.; Cassimatis, T.M.; Kelsey, N.; et al. Chronic mirabegron treatment increases human brown fat, HDL cholesterol, and insulin sensitivity. J. Clin. Investig. 2020, 130, 2209-2219. https://doi.org/10.1172/JCI131126.

11. Finlin, B.S.; Memetimin, H.; Zhu, B.; Confides, A.L.; Vekaria, H.J.; El Khouli, R.H.; Johnson, Z.R.; Westgate, P.M.; Chen, J.; Morris, A.J.; et al. The beta3-adrenergic receptor agonist mirabegron improves glucose homeostasis in obese humans. J. Clin. Investig. 2020, 130, 2319-2331. https://doi.org/10.1172/JCI134892.

12. Gil-Ortega, M.; Somoza, B.; Huang, Y.; Gollasch, M.; Fernandez-Alfonso, M.S. Regional differences in perivascular adipose tissue impacting vascular homeostasis. Trends Endocrinol. Metab. 2015, 26, 367-375. https://doi.org/10.1016/j.tem.2015.04.003.

13. Chang, L.; Villacorta, L.; Li, R.; Hamblin, M.; Xu, W.; Dou, C.; Zhang, J.; Wu, J.; Zeng, R.; Chen, Y.E. Loss of perivascular adipose tissue on peroxisome proliferator-activated receptor-gamma deletion in smooth muscle cells impairs intravascular thermoregulation and enhances atherosclerosis. Circulation 2012, 126, 1067-1078. https://doi.org/10.1161/CIRCULATIONAHA.112.104489. 
14. Sui, W.; Li, H.; Yang, Y.; Jing, X.; Xue, F.; Cheng, J.; Dong, M.; Zhang, M.; Pan, H.; Chen, Y.; et al. Bladder drug mirabegron exacerbates atherosclerosis through activation of brown fat-mediated lipolysis. Proc. Natl. Acad. Sci. USA 2019, 116, 10937-10942. https://doi.org/10.1073/pnas.1901655116.

15. Raun, S.H.; Olguín, C.H.; Karavaeva, I.; Ali, M.; Møller, L.L.V.; Kot, W.; Castro Mejía, J.L.; Nielsen, D.S.; Gerhart-Hines, Z.; Richter, E.A.; et al. Housing temperature influences exercise training adaptations in mice. Nat. Commun. 2020, 11, 1560.

16. McKie, G.L.; Medak, K.D.; Knuth, C.M.; Shamshoum, H.; Townsend, L.K.; Peppler, W.T.; Wright, D.C. Housing temperature affects the acute and chronic metabolic adaptations to exercise in mice. J. Physiol. 2019, 597, 4581-4600. https://doi.org/10.1113/JP278221.

17. Clayton, Z.S.; McCurdy, C.E. Short-term thermoneutral housing alters glucose metabolism and markers of adipose tissue browning in response to a high-fat diet in lean mice. Am. J. Physiol. Regul. Integr. Comp. Physiol. 2018, 315, R627-R637. https://doi.org/10.1152/ajpregu.00364.2017.

18. Hylander, B.L.; Repasky, E.A. Thermoneutrality, Mice, and Cancer: A Heated Opinion. Trends Cancer 2016, 2, 166-175. https://doi.org/10.1016/j.trecan.2016.03.005.

19. Rowland, L.A.; Maurya, S.K.; Bal, N.C.; Kozak, L.; Periasamy, M. Sarcolipin and uncoupling protein 1 play distinct roles in dietinduced thermogenesis and do not compensate for one another. Obesity 2016, 24, 1430-1433. https://doi.org/10.1002/oby.21542.

20. Stern, J.S.; Inokuchi, T.; Castonguay, T.W.; Wickler, S.J.; Horwitz, B.A. Scapular brown fat removal enhances development of adiposity in cold-exposed obese Zucker rats. Am. J. Physiol. 1984, 247 Pt 2, R918-R926. https://doi.org/10.1152/ajpregu.1984.247.5.R918.

21. Yoo, H.S.; Qiao, L.; Bosco, C.; Leong, L.H.; Lytle, N.; Feng, G.S.; Chi, N.W.; Shao, J. Intermittent cold exposure enhances fat accumulation in mice. PLOS ONE 2014, 9, e96432. https://doi.org/10.1371/journal.pone.0096432.

22. Young, J.B.; Shimano, Y. Effects of rearing temperature on body weight and abdominal fat in male and female rats. Am. J. Physiol. 1998, 274, R398-R405. https://doi.org/10.1152/ajpregu.1998.274.2.R398.

23. Nilaweera, K.N.; Speakman, J.R. Regulation of intestinal growth in response to variations in energy supply and demand. Obes. Rev. 2018, 19 (Suppl. S1), 61-72. https://doi.org/10.1111/obr.12780.

24. Price, E.R.; Ruff, L.J.; Guerra, A.; Karasov, W.H. Cold exposure increases intestinal paracellular permeability to nutrients in the mouse. J. Exp. Biol. 2013, 216(Pt 21), 4065-4070. https://doi.org/10.1242/jeb.088203.

25. Chevalier, C.; Stojanovic, O.; Colin, D.J.; Suarez-Zamorano, N.; Tarallo, V.; Veyrat-Durebex, C.; Rigo, D.; Fabbiano, S.; Stevanovic, A.; Hagemann, S.; et al. Gut Microbiota Orchestrates Energy Homeostasis during Cold. Cell 2015, 163, 1360-1374. https://doi.org/10.1016/j.cell.2015.11.004.

26. Fischer, A.W.; Csikasz, R.; von Essen, G.; Cannon, B.; Nedergaard, J. No insulating effect of obesity. Am. J. Physiol. Endocrinol. Metab. 2016, 311, E202-E213. https://doi.org/10.1152/ajpendo.00093.2016.

27. Jay, O.; Raubenheimer, D. Some problems with translating the insulating effect of obesity from mice to men. Am. J. Physiol. Endocrinol. Metab. 2016, 311, E638. https://doi.org/10.1152/ajpendo.00265.2016.

28. Fischer, A.W.; Csikasz, R.I.; von Essen, G.; Cannon, B.; Nedergaard, J. Reply to letter to the editor: At thermoneutrality, neither the lean nor the obese freeze. Am. J. Physiol. Endocrinol. Metab. 2016, 311, E639. https://doi.org/10.1152/ajpendo.00309.2016.

29. Tang, Q.Q.; Otto, T.C.; Lane, M.D. Mitotic clonal expansion: A synchronous process required for adipogenesis. Proc. Natl. Acad. Sci. USA 2003, 100, 44-49. https://doi.org/10.1073/pnas.0137044100.

30. Merkestein, M.; Laber, S.; McMurray, F.; Andrew, D.; Sachse, G.; Sanderson, J.; Li, M.; Usher, S.; Sellayah, D.; Ashcroft, F.M.; et al. FTO influences adipogenesis by regulating mitotic clonal expansion. Nat. Commun. 2015, 6, 6792. https://doi.org/10.1038/ncomms7792.

31. Hou, X.; Zhang, Y.; Li, W.; Hu, A.J.; Luo, C.; Zhou, W.; Hu, J.K.; Daniele, S.G.; Wang, J.; Sheng, J.; et al. CDK6 inhibits white to beige fat transition by suppressing RUNX1. Nat. Commun. 2018, 9, 1023. https://doi.org/10.1038/s41467-018-03451-1.

32. Vishvanath, L.; Gupta, R.K. Contribution of adipogenesis to healthy adipose tissue expansion in obesity. J. Clin. Investig. 2019, 129, 4022-4031. https://doi.org/10.1172/JCI129191.

33. Loh, R.K.C.; Formosa, M.F.; La Gerche, A.; Reutens, A.T.; Kingwell, B.A.; Carey, A.L. Acute metabolic and cardiovascular effects of mirabegron in healthy individuals. Diabetes Obes. Metab. 2019, 21, 276-284. https://doi.org/10.1111/dom.13516.

34. Baskin, A.S.; Linderman, J.D.; Brychta, R.J.; McGehee, S.; Anflick-Chames, E.; Cero, C.; Johnson, J.W.; O'Mara, A.E.; Fletcher, L.A.; Leitner, B.P.; et al. Regulation of Human Adipose Tissue Activation, Gallbladder Size, and Bile Acid Metabolism by a beta3-Adrenergic Receptor Agonist. Diabetes 2018, 67, 2113-2125. https://doi.org/10.2337/db18-0462.

35. Cypess, A.M.; Weiner, L.S.; Roberts-Toler, C.; Franquet Elia, E.; Kessler, S.H.; Kahn, P.A.; English, J.; Chatman, K.; Trauger, S.A.; Doria, A.; et al. Activation of human brown adipose tissue by a beta3-adrenergic receptor agonist. Cell Metab. 2015, $21,33-38$. https://doi.org/10.1016/j.cmet.2014.12.009.

36. Finlin, B.S.; Memetimin, H.; Confides, A.L.; Kasza, I.; Zhu, B.; Vekaria, H.J.; Harfmann, B.; Jones, K.A.; Johnson, Z.R.; Westgate, P.M.; et al. Human adipose beiging in response to cold and mirabegron. JCI Insight 2018, 3, e121510. https://doi.org/10.1172/jci.insight.121510.

37. Aizawa, N.; Homma, Y.; Igawa, Y. Effects of mirabegron, a novel beta3-adrenoceptor agonist, on primary bladder afferent activity and bladder microcontractions in rats compared with the effects of oxybutynin. Eur. Urol. 2012, 62, 1165-1173. https://doi.org/10.1016/j.eururo.2012.08.056. 
38. Hatanaka, T.; Ukai, M.; Watanabe, M.; Someya, A.; Ohtake, A.; Suzuki, M.; Ueshima, K.; Sato, S.; Kaku, S. Effect of mirabegron, a novel beta3-adrenoceptor agonist, on bladder function during storage phase in rats. Naunyn Schmiedebergs Arch. Pharm. 2013, 386, 71-78. https://doi.org/10.1007/s00210-012-0814-3.

39. Sacco, E.; Bientinesi, R.; Tienforti, D.; Racioppi, M.; Gulino, G.; D’Agostino, D.; Vittori, M.; Bassi, P. Discovery history and clinical development of mirabegron for the treatment of overactive bladder and urinary incontinence. Expert Opin. Drug Discov. 2014, 9, 433-448. https://doi.org/10.1517/17460441.2014.892923.

40. Takasu, T.; Ukai, M.; Sato, S.; Matsui, T.; Nagase, I.; Maruyama, T.; Sasamata, M.; Miyata, K.; Uchida, H.; Yamaguchi, O. Effect of (R)-2-(2-aminothiazol-4-yl)-4'-[1] acetanilide (YM178), a novel selective beta3-adrenoceptor agonist, on bladder function. J. Pharmacol. Exp. Ther. 2007, 321, 642-647. https://doi.org/10.1124/jpet.106.115840.

41. Au-Yong, I.T.; Thorn, N.; Ganatra, R.; Perkins, A.C.; Symonds, M.E. Brown adipose tissue and seasonal variation in humans. Diabetes 2009, 58, 2583-2587. https://doi.org/10.2337/db09-0833.

42. Gelinas, J.N.; Banko, J.L.; Hou, L.; Sonenberg, N.; Weeber, E.J.; Klann, E.; Nguyen, P.V. ERK and mTOR signaling couple betaadrenergic receptors to translation initiation machinery to gate induction of protein synthesis-dependent long-term potentiation. J. Biol. Chem. 2007, 282, 27527-27535. https://doi.org/10.1074/jbc.M701077200.

43. Hostrup, M.; Reitelseder, S.; Jessen, S.; Kalsen, A.; Nyberg, M.; Egelund, J.; Kreiberg, M.; Kristensen, C.M.; Thomassen, M.; Pilegaard, H.; et al. Beta2-adrenoceptor agonist salbutamol increases protein turnover rates and alters signalling in skeletal muscle after resistance exercise in young men. J. Physiol. 2018, 596, 4121-4139. https://doi.org/10.1113/JP275560.

44. Puzzo, D.; Raiteri, R.; Castaldo, C.; Capasso, R.; Pagano, E.; Tedesco, M.; Gulisano, W.; Drozd, L.; Lippiello, P.; Palmeri, A.; et al. CL316,243, a beta3-adrenergic receptor agonist, induces muscle hypertrophy and increased strength. Sci. Rep. 2016, 5, 37504. https://doi.org/10.1038/srep37504.

45. Emery, P.W.; Rothwell, N.J.; Stock, M.J.; Winter, P.D. Chronic effects of beta 2-adrenergic agonists on body composition and protein synthesis in the rat. Biosci. Rep. 1984, 4, 83-91. https://doi.org/10.1007/bf01120827.

46. Young, J.B.; Weiss, J.; Boufath, N. Effects of rearing temperature on sympathoadrenal activity in young adult rats. Am. J. Physiol. Regul. Integr. Comp. Physiol. 2002, 283, R1198-R1209. https://doi.org/10.1152/ajpregu.00525.2001.

47. White, C.L.; Braymer, H.D.; York, D.A.; Bray, G.A. Effect of a high or low ambient perinatal temperature on adult obesity in Osborne-Mendel and S5B/Pl rats. Am. J. Physiol. Regul. Integr. Comp. Physiol. 2005, 288, R1376-R1384. https://doi.org/10.1152/ajpregu.00162.2004.

48. Hawkins, P.; Golledge, H.D.R. The 9 to 5 Rodent-Time for Change? Scientific and animal welfare implications of circadian and light effects on laboratory mice and rats. J. Neurosci. Methods 2018, 300, 20-25. https://doi.org/10.1016/j.jneumeth.2017.05.014.

49. Aldiss, P.; Lewis, J.; Boocock, D.; Miles, A.; Bloor, I.; Ebling, F.; Budge, H.; Symonds, M. Interscapular and Perivascular Brown Adipose Tissue Respond Differently to a Short-Term High-Fat Diet. Nutrients 2019, 11, 1065.

50. Galarraga, M.; Campion, J.; Munoz-Barrutia, A.; Boque, N.; Moreno, H.; Martinez, J.A.; Milagro, F.; Ortiz-de-Solorzano, C. Adiposoft: Automated software for the analysis of white adipose tissue cellularity in histological sections. J. Lipid Res. 2012, 53, 2791-2796. https://doi.org/10.1194/jlr.D023788.

51. Mele, L.; Paino, F.; Papaccio, F.; Regad, T.; Boocock, D.; Stiuso, P.; Lombardi, A.; Liccardo, D.; Aquino, G.; Barbieri, A.; et al. A new inhibitor of glucose-6-phosphate dehydrogenase blocks pentose phosphate pathway and suppresses malignant proliferation and metastasis in vivo. Cell Death Dis. 2018, 9, 572. https://doi.org/10.1038/s41419-018-0635-5.

52. Lambert, J.P.; Ivosev, G.; Couzens, A.L.; Larsen, B.; Taipale, M.; Lin, Z.Y.; Zhong, Q.; Lindquist, S.; Vidal, M.; Aebersold, R.; et al. Mapping differential interactomes by affinity purification coupled with data-independent mass spectrometry acquisition. Nat. Methods 2013, 10, 1239-1245. https://doi.org/10.1038/nmeth.2702.

53. Ashburner, M.; Ball, C.A.; Blake, J.A.; Botstein, D.; Butler, H.; Cherry, J.M.; Davis, A.P.; Dolinski, K.; Dwight, S.S.; Eppig, J.T.; et al. Gene ontology: Tool for the unification of biology. The Gene Ontology Consortium. Nat. Genet. 2000, $25,25-29$. https://doi.org/10.1038/75556.

54. Alexa, A.; Rahnenfuhrer, J.; Lengauer, T. Improved scoring of functional groups from gene expression data by decorrelating GO graph structure. Bioinformatics 2006, 22, 1600-1607. https://doi.org/10.1093/bioinformatics/btl140. 